\title{
Low-energy interactions related to atmospheric and extreme conditions $^{\star}$
}

\author{
Sylwia Ptasinska ${ }^{1, a}$, Małgorzata A. Śmiałek ${ }^{2,3}$, Aleksandar R. Milosavljević ${ }^{4,5}$, \\ and Bhalamurugan Sivaraman ${ }^{3,6}$ \\ ${ }^{1}$ Radiation Laboratory and Department of Physics, University of Notre Dame, Notre Dame, IN 46556, USA \\ ${ }^{2}$ Gdansk University of Technology, Department of Control and Power Engineering, Faculty of Ocean Eng. and \\ Ship Technology, Gdańsk, Poland \\ 3 School of Physical Sciences, The Open University, Milton Keynes, UK \\ ${ }^{4}$ SOLEIL, l'Orme des Merisiers, St Aubin, BP48, 91192 Gif sur Yvette Cedex, France \\ ${ }^{5}$ University of Belgrade, Institute of Physics Belgrade, Laboratory for Atomic Collision Processes, Beograd, Serbia \\ 6 Atomic, Molecular and Optical Physics Division, Physical Research Laboratory, Ahmedabad, India
}

Received 8 August 2017

Published online 19 October 2017 - (c) EDP Sciences, Società Italiana di Fisica, Springer-Verlag 2017

This Topical Issue, entitled "Low-Energy Interactions Related to Atmospheric and Extreme Conditions", showcases a collection of eighteen articles that reported recent theoretical and experimental findings pertaining to the following topics:

- low-energy interactions of charged particles (electrons [1-7], protons [8], positrons [9]), and photons [10] with atoms and molecules of biological [1-4,7,8], astrochemical [10], industrial, and environmental $[5,6,9]$ interest;

- molecular evolution of astrochemical ices [11-13];

- planetary atmospheric physics [14];

- atmospheric pressure photoionization and fragmentation of organic and inorganic aerosols [15];

- processes and applications of atmospheric pressure plasmas in astrochemical [16,17] and biomedical fields $[17,18]$.

The contributed articles revealed that, in recent years, processes involving any kind of low-energy projectile (below a few keV) over a wide range of conditions have been recognized as important to many interdisciplinary research areas that span both the fundamental and applied sciences. For example, many studies on the topic of low-energy interactions (LEIs) have focused on probing gas or condensed phases under high-vacuum conditions. These studies model processes that can occur under physiological, industrial, atmospheric, or extreme conditions, the latter of which represent those that experience high levels of radiation in space. Therefore, this issue

\footnotetext{
${ }^{\star}$ Contribution to the Topical Issue "Low-Energy Interactions related to Atmospheric and Extreme Conditions", edited by S. Ptasinska, M. Smialek-Telega, A. Milosavljevic, B. Sivaraman.

a e-mail: sptasins@nd.edu
}

focuses on the most recent advances in the field of lowenergy processes, both at the fundamental and applied levels of elementary processes, and provides a state-of-theart description of current developments and discoveries. What follows is a brief summary of the most important conclusions derived from the collective findings.

Over the last two decades, many LEI studies have focused on isolated biomolecules in the gas and condensed phases to understand and predict more accurately the reaction pathways and detailed mechanisms of damage to cellular components, such as DNA and proteins. In this issue, Śmiałek summarized and highlighted the pioneering work, together with the most prominent findings about DNA damage from an atomic and molecular physics perspective; this timely discussion contributes to a deeper understanding of the fundamental processes in cellular systems [7]. In her colloquium paper, Śmiałek also discussed experimental issues and challenges experienced in the precise estimation of cross sections for DNA damage [7]. Similarly, in another contributed article, Rackwitz et al. discussed the difficulties in establishing a reliable cross section for DNA damage, particularly strand breaks [3]. To overcome these challenges, the authors proposed a novel method to quantify absolute cross sections of strand breaks that low-energy electrons induce in oligonucleotides (synthesized, single-stranded DNA). Using this technique, oligonucleotides (with and without radiosensitizers) were supported on a DNA origami triangle and irradiated with $10 \mathrm{eV}$ electrons. Thereafter, the cross sections of damage were determined based on the analysis of atomic force microscopic images [3].

In addition to Śmiałek's summary of the current state of our understanding of DNA damage [7], other contributed articles reported recent experimental results for other biologically-important molecules, primarily amino 
acids that can act as protein model compounds. These works focused on fragmentation of cysteine [1] and proline [2] induced by low-energy electrons through the processes of dissociative electron attachment and electron impact ionization, respectively. Alizadeh et al. demonstrated ion desorption yields from physisorbed and chemisorbed cysteine films irradiated by electrons [1] and measured using mass spectrometry. There is a particular interest in the latter deposition method in biomedical applications, such as radiotherapy in combination with gold nanoparticles and in organic sensors. This study showed that when cysteine is chemisorbed through the thiol group onto a clean gold substrate, ion yields of hydroxyl anions increased; this also held true for heavier fragments when compared to desorption yields from physisorbed films [1]. Mass spectrometry also was used to investigate fragmentation of another amino acid, proline [2]; however, in contrast to the cysteine work, this study focused on cation formation from a gas-phase molecule. Tamuliene et al. reported the ionization energy of proline and the appearance energies of the main cationic fragments produced in LEIs [2]. The authors supported their fragment assignment by calculating the appearance energies of different proline conformers. Moreover, they determined the possible dissociation mechanism of the dominant fragmentation pathway, in which one electron is removed from the lone pair of the nitrogen atom, and results eventually in the cleavage of the $\mathrm{C}-\mathrm{C}_{\alpha}$ bond accompanied by the loss of $\mathrm{COOH}[2]$. A theoretical investigation of total cross sections of electron impact in a wide energy range, from the ionization threshold to $5 \mathrm{keV}$, also was performed for tetrahydrofuran [4]. This molecule often is used as a model for the DNA sugar deoxyribose, and recently has been studied extensively by experimental and theoretical research groups. The theoretical data by Swadia et al. employed spherical complex optical potential and complex scattering potential-ionization contribution formalisms to compute several types of total cross sections [4]. The results correlated well with data available in the extant literature; therefore, the authors will use the proposed calculation method for other complex biomolecules in the future. LEIs with furan, a simpler analogue of tetrahydrofuran, also were reported in one of the contributed articles by Wasowicz et al. [8]. However, the authors used a proton beam as an incident projectile, in contrast to the aforementioned electron-collision studies, marking the first report on the investigation of protoninduced dissociation of furan by measuring the photon emission spectra of the excited fragments [8]. Notably, the emission intensities recorded at different proton energies showed the highest yield for a hydrogen atom, which decreased as a function of the principal quantum number of the excited $\mathrm{H}$ atoms. These experimental data also were compared to the emission spectra data obtained from collisions of proton, oxygen, and carbon cations with tetrahydrofuran [8]. Based on these data, the authors suggested possible mechanisms for the enhanced hydrogen production [8].

In addition to the many studies of charged projectiles with biomolecules, a great focus has been placed on LEIs with atoms and molecules. This issue also contains three theoretical articles about electron impact excitation of several highly-charged tungsten (W) ions [5], tin (Sn) [6], and positron scattering with two atomic targets (argon and krypton) and two molecular targets (nitrogen and methane) [9]. The electron impact excitation of $\mathrm{W}$ and $\mathrm{Sn}$ are particularly important to the field of plasma, in which data on excited atoms and ions are needed to develop realistic plasma models. Priti et al. employed the relativistic distorted wave (RDW) approximation theory to calculate the cross sections of $\mathrm{W}^{37+}, \mathrm{W}^{38+}$, and $\mathrm{W}^{39+}$ for different transitions induced by electrons in a wide energy range up to $20 \mathrm{keV}$ [5]. The RDW method was also employed to calculate electron impact excitation in a tin atom [6]. In the case of Sn excitation, Sharma et al. obtained differential cross sections at several incident electron energies $(20,50,80$, and $100 \mathrm{eV})$ and integrated cross sections for the electron energy range between 5 and $100 \mathrm{eV}$ [6]. They compared the results for both $\mathrm{W}$ and Sn excitation energies and oscillator strengths to those published previously to verify the accuracy of their method $[5,6]$. Moreover, Priti et al. also attempted to use analytical formulas to fit the cross sections they obtained, which can be applied directly in plasma modeling [5]. In Franz' article, the author recommended using correlation-polarization potentials with the quantum Monte Carlo method to calculate scattering cross sections of positrons with atoms and molecules [9]. These potentials can be represented using simple analytical formulas and can be implemented easily in existing computer programs. To validate the suggested quantum Monte Carlo method of obtaining positron-electron correlation-polarization potentials, this approach also was used for positron scattering from Ar, $\mathrm{Kr}, \mathrm{N}_{2}$, and $\mathrm{CH}_{4}$ as examples [9].

The interactions of charged projectiles with organic molecules were not the only interactions investigated. Vacuum ultraviolet (VUV) photons were used to probe electronic transitions, in ethyl acetate $\left(\mathrm{C}_{4} \mathrm{H}_{8} \mathrm{O}_{2}\right)$ [10], e.g. Śmiałek et al. reported the complete electronic spectra of this molecule, and assigned its band structures based on ab initio calculations. Further, they provided the absolute photoabsorption cross sections from 4.5 to $10.7 \mathrm{eV}$ [10]. $\mathrm{A}_{4} \mathrm{H}_{8} \mathrm{O}_{2}$ compound belongs to a group of esters, an important class of oxygenated volatile organic compounds. Understanding atmospheric degradation of esters is crucial because of their contribution to ecological effects. Therefore, based on these cross sections, the authors calculated the photolysis lifetime of ethyl acetate in the Earth's troposphere and stratosphere. A comparison of rate constants for the reactions of ethyl acetate with several radicals indicated that its atmospheric photolysis is negligible [10]. Sharma et al.'s article investigated atmospheric temperatures at high altitudes in western India [14]. They combined satellite observations with computational models to expand existing knowledge of various geophysical and atmospheric processes [14].

The article by Baeza-Romero et al. proposed the use of a new instrument, a smog chamber in which a photoionization aerosol electron/ion spectrometer is coupled with VUV synchrotron radiation [15]. Organic aerosols are known to affect atmospheric processes significantly, and thus, human health. This newly developed instrument was tested for on-line analysis of these organic and 
inorganic/organic mixed aerosols [15]. Several interesting results were presented, for example, the ozonolysis of $\alpha$-pinene and the uptake of glyoxal by ammonium sulfate [15]. The authors monitored the concentration of ozone in real time, together with particle size distribution and chemical composition. They also discussed further improvement of this instrumentation [15].

Switching from investigations of atmospheric conditions to extreme conditions that correspond to those present in an interstellar medium, three theoretical works were contributed to this issue. These articles pertained to the chemical composition of the interstellar medium, meteorites, and grain mantles. Etim et al. employed high-level quantum chemical calculations of a $\mathrm{C}_{5} \mathrm{H}_{9} \mathrm{~N}$ isomeric group to verify the stability of these isomers [12]. Their calculations showed that one of the isomers, n-butyl cyanide, which was not detected in astrochemical observations, is unstable, which might be one reason that this isomer was not observed. The authors proposed another isomer, tert-butylnitrile, as a target for astrochemical search, as this molecule is more stable and its expected intensity ratio, assuming the same column density and rotational temperature, is the maximum among this isomeric group [12]. The authors also performed local thermodynamic equilibrium modeling to verify the possibility of detecting this isomer in the millimeter-wave region. To study the chemical compositions of interstellar media, Shivani et al. used a different approach, in which radical-neutral interactions led to the formation of biological compounds [13]. They investigated the reaction pathways, which involved interactions of hydrogenated methyl cyanide $\left(\mathrm{CH}_{3} \mathrm{CNH}^{+}\right)$with formic acid $(\mathrm{HCOOH})$ that resulted in alanine formation, an amino acid detected in astrochemical observations. This reaction was calculated using quantum chemical methods involving density functional theory at the B3LYP/6-311G $(\mathrm{d}, \mathrm{p})$ level [13]. The results indicated that the reaction was exothermic with a small potential barrier and, thus, is likely to occur in interstellar space [13]. Sil et al. also used quantum chemical calculations to investigate the adsorption energies of $\mathrm{H}$ and $\mathrm{H}_{2}$ [11]. Because hydrogen, in either atomic or molecular form, is the most common element in space, the authors attempted to calculate its binding energies to several adsorbents (benzene as a simple unit of polycyclic aromatic hydrocarbons, and silicon dioxide as a model of silicate grain), which also included water clusters [11]. Their results showed that the binding energies of atomic hydrogen are lower than are those for molecular hydrogen. Interestingly, experimental data reported previously have shown the opposite trend in some cases [11].

The remaining articles focused on atmospheric pressure plasmas for astrochemical $[16,17]$ and medical $[17,18]$ applications. Although the articles mentioned previously also addressed these types of applications, they did so with LEIs of single projectiles and molecules or atoms. Plasma interactions are more complex because plasma contains not only charged particles, but excited neutral radicals and photons as well. Sahoo et al. employed the equation of motion and Fock-space coupled cluster methods to obtain ionization potentials and excitation energies for magnesium and iron ions (He-like and Li-like) in a plasma environment [16]. These calculations focused on the transition lines for the ions with ion and electron densities on the order of $10^{21} \mathrm{~cm}^{-3}$ in the temperature range of $0-150 \mathrm{eV}$. They compared their results with the data available in the literature, and found strong concurrence. The authors also noted different trends in ionization potential and electron affinity change for He-like and Lilike ions, and discussed other properties of such systems [16]. These results can be included in plasma models and potentially could be helpful in interpreting laboratory and astrophysical plasmas.

Čech et al. performed a mass spectrometric study designed to characterize diffuse coplanar surface barrier discharge at atmospheric pressures [17]. The authors detected fragments produced from different gas mixtures of $\mathrm{N}_{2}$ and $\mathrm{O}_{2}$, with special attention to the formation of the ozone and nitric oxide molecules. These two molecules were selected as representative compounds to observe the effects of different plasma parameters (i.e., gas mixture flow rate and driving frequency) while other parameters were kept constant [17]. The authors also selected two molecules, $\mathrm{O}_{3}$ and $\mathrm{NO}$, because they play an important role in biological systems [17]. Adhikari et al. investigated the total effects of plasma species in atmospheric pressure plasma jets [18]. In their work, helium atmospheric pressure plasma was used to cause strand breaks in DNA. The DNA molecules acted as probes to investigate different parameters of plasma (He flow rate, plasma irradiation time, distance between the plasma and a sample, DNA concentration, and applied voltage). The goal of this study was to determine the correlation between experimental parameters and DNA damage to identify the optimal experimental setting for biomedical applications desired.

The editors would like to thank all of the contributing authors for their significant input in creating this Topical Issue and for sharing their valuable data, which will improve our understanding significantly and advance experimental and theoretical studies in the field of lowenergy interactions related to atmospheric and extreme conditions.

S.P. acknowledges the U.S. Department of Energy Office of Science, Office of Basic Energy Sciences under Award Number DE-FC02-04ER15533. This is contribution number NDRL 5185 from the Notre Dame Radiation Laboratory. A.R.M. acknowledges the support from MESTD of Republic of Serbia (\#171020). B.S. would like to acknowledge the support from INSPIRE Grant (IFA-11CH-11) and Physical Research Laboratory.

\section{Author contribution statement}

All authors contributed to writing this editorial equally.

\section{References}

1. E. Alizadeh, S. Massey, L. Sanche, P.A. Rowntree, Eur. Phys. J. D 70, 75 (2016) 
2. J. Tamuliene, L. Romanova, V. Vukstich, A. Papp, S. Shkurin, L. Baliulyte, A. Snegursky, Eur. Phys. J. D 70, 143 (2016)

3. J. Rackwitz, M.L. Ranković, A.R. Milosavljević, I. Bald, Eur. Phys. J. D 71, 32 (2017)

4. M. Swadia, Y. Thakar, M. Vinodkumar, C. Limbachiya, Eur. Phys. J. D 71, 85 (2017)

5. Priti, L. Sharma, R. Srivastava, Eur. Phys. J. D 71, 100 (2017)

6. L. Sharma, S. Bharti, R. Srivastava, Eur. Phys. J. D 71, 121 (2017)

7. M.A. Śmiałek, Eur. Phys. J. D 70, 237 (2016)

8. T.J. Wasowicz, B. Pranszke, Eur. Phys. J. D 70, 175 (2016)

9. J. Franz, Eur. Phys. J. D 71, 44 (2017)

10. M.A. Smialek, M. Labuda, J. Guthmuller, M.-J. Hubin-Franskin, J. Delwiche, S.V. Hoffmann, N.C. Jones,
N.J. Mason, P. Limao-Viera, Eur. Phys. J. D 70, 138 (2016)

11. M. Sil, P. Gorai, A. Das, D. Sahu, S.K. Chakrabarti, Eur. Phys. J. D 71, 45 (2017)

12. E. Etim, P. Gorai, A. Das, E. Arunan, Eur. Phys. J. D 71, $86(2017)$

13. Shivani, P. Pandey, A. Misra, P. Tandon, Eur. Phys. J. D 71, 215 (2017)

14. S. Sharma, R. Vaishnav, K.K. Shukla, S. Lal, H. Chandra, Y.B. Acharya, Eur. Phys. J. D 71, 187 (2017)

15. M.T. Baeza-Romero, F. Gaie-Levrel, A. Mahjoub, V. López-Arza, G.A. Garcia Gustavo, L.A. Nahon, Eur. Phys. J. D 70, 154 (2016)

16. B.K. Sahoo, M. Das, Eur. Phys. J. D 70, 270 (2016)

17. J. Čech, A. Brablec, M. Černak, N. Puač, N. Selakovič, Z.L. Petrovič, Eur. Phys. J. D 71, 27 (2017)

18. E.R. Adhikari, S. Ptasinska, Eur. Phys. J. D 70, 180 (2016) 